\title{
Chemical equilibration in weakly coupled QCD
}

\author{
Aleksi Kurkela* \\ Theoretical Physics Department, CERN, Geneva, Switzerland and Faculty of Science and Technology, \\ University of Stavanger, 4036 Stavanger, Norway \\ Aleksas Mazeliauskas ${ }^{\dagger}$ \\ Institut für Theoretische Physik, Universität Heidelberg, 69120 Heidelberg, Germany
}

(Received 8 November 2018; published 25 March 2019)

\begin{abstract}
We study thermalization, hydrodynamization, and chemical equilibration in out-of-equilibrium quarkgluon plasma starting from various initial conditions using QCD effective kinetic theory, valid at weak coupling. In nonexpanding systems gauge bosons rapidly lose information of the initial state and achieve kinetic equilibrium among themselves, while fermions approach the equilibrium distribution only at a later time. In systems undergoing rapid longitudinal expansion, both gluons and quarks are kept away from equilibrium by the expansion, but the evolution is well described by fluid dynamics even before local thermal equilibrium is reached. For realistic couplings we determine the ordering between the separate hydrodynamization, chemical equilibration and thermalization time scales to be $\tau_{\text {hydro }}<\tau_{\text {chem }}<\tau_{\text {therm }}$.
\end{abstract}

DOI: $10.1103 /$ PhysRevD.99.054018

\section{INTRODUCTION}

How gauge theories pushed far from equilibrium thermalize is a central topic in the study of heavy-ion collisions [1]. To what extent the postcollisional debris created in the collision of two nuclei reaches local thermal equilibrium before the system cools down determines how well a fluid dynamical description of the system is applicable.

Our ability to perform first principles nonperturbative real-time calculations in QCD is limited by the infamous sign problem [2], and considerable efforts have been invested to understand thermalization and hydrodynamization in various approximations of the QCD. A prominent example is the $\mathcal{N}=4$ super Yang-Mills theory in the limit of a large number of colors and strong coupling, which has been studied extensively with holographic methods [3-6]. The holographic methodology can be applied only to a very limited set of gauge theories, and for generic theoriessuch as QCD—only weak-coupling methods are available. So far the weak-coupling studies of thermalization of farfrom-equilibrium systems have been limited to either pure gauge or scalar theories, and studies in QCD have been restricted only to near-equilibrium systems $[7,8]$. Here,

\footnotetext{
a.k@cern.ch

†.mazeliauskas@thphys.uni-heidelberg.de
}

Published by the American Physical Society under the terms of the Creative Commons Attribution 4.0 International license. Further distribution of this work must maintain attribution to the author(s) and the published article's title, journal citation, and DOI. Funded by SCOAP . we extend the weak-coupling treatment of $[9,10]$ by including dynamical fermions and study how far-fromequilibrium systems approach equilibrium in a full leading order QCD description.

Introducing new degrees of freedom (d.o.f.) to the system adds new structures. It has been argued [11] that the off-equilibrium dynamics of quarks may be significantly slower than that of the gluons, owing partly to smaller group theoretic color factors and partly to different spin statistics and Pauli blocking. It may be then that the equilibration of quarks could be a bottleneck of thermalization as chemical equilibration may take place in a significantly longer timescale. In particular, in the weakcoupling picture of heavy-ion collisions, the initial state in midrapidity is dominated by a large number of gluons with only a few fermions. If the production of fermions is delayed, this could have an impact on the fluid dynamical modeling of heavy-ion collisions, since the equation of state of quark-gluon plasma (QGP) is different than that of plasma consisting of gluons only. Furthermore, chemically equilibrated QGP is a standard explanation of the strangeness enhancement in nucleus-nucleus collisions [12-14], so understanding fermion production from first principles provides an important theoretical validation of this picture.

It has been observed in several theories-both weakly and strongly coupled - that the hydrodynamical constitutive relations are approximately fulfilled in systems that have sizable anisotropies, that is, hydrodynamization without thermalization $[5,10,15,16]$. Upon including quark d.o.f. to the system we may ask the question, when does the chemical equilibration happen with respect to the 
hydrodynamization and thermalization times $\tau_{\text {hydro }}$ and $\tau_{\text {therm }}$ ?

The basic tool here is the set of Boltzmann equations that are applicable in isotropic systems any time the typical occupancies are smaller than $1 / \alpha_{s}$. Of course, many authors have already considered the evolution of quarkgluon systems under Boltzmann equations [17-25]. What sets our study apart from these works is that we use the effective Boltzmann equations derived by Arnold, Moore, and Yaffe [26], which account for all processes needed for a description that is accurate to leading order in $\alpha_{s}$. These processes include in-medium screening effects [27] and Landau-Pomeranchuk-Migdal [28-31] corrected splitting processes in far-from-equilibrium but isotropic systems. ${ }^{1}$ To do so we extend the previously developed setup of Refs. $[6,9,10,33]$ to include quark d.o.f. and we briefly summarize the key elements of the description in Sec. II.

In the following we will consider $N_{c}=3 \mathrm{QCD}$ plasma with $N_{f}=3$ flavors of massless quarks in different out-ofequilibrium conditions, with and without expansion. While there is only one thermal equilibrium, there are many ways a system can be out of equilibrium. In Sec. III A we will first study in detail a particularly simple nonexpanding system where quarks are absent and gluons are in kinetic equilibrium among themselves. We discuss the processes that produce fermions and subsequently lead to chemical equilibration. In Sec. III B we then move on to discuss nonexpanding systems which are initialized with an overoccupied gauge boson distribution. While we are unaware of a physical system where QCD would be found in these conditions, cosmological reheating may result in a system of overoccupied gauge bosons (see e.g., [34]). Following the time evolution of the overoccupied system, we see that due to the slower dynamics of fermions, gauge bosons reach kinetic equilibrium before chemical equilibration. Once the gauge bosons have reached kinetic equilibrium the evolution proceeds as in our first example. Finally, in Sec. IV we turn to a system of overoccupied gluons undergoing boost-invariant longitudinal expansion. This is the expected initial condition in heavy-ion collisions in the asymptotic weak-coupling limit $[35,36]$. For moderate values of the coupling constant $\alpha_{s} \sim 0.3$ we observe a rapid memory loss of initial conditions and chemical and hydrodynamical equilibrium is approached along a universal curve. Finally, we conclude with the discussion of the separate equilibration timescales in Sec. V.

\section{EFFECTIVE KINETIC THEORY}

The effective kinetic theory that we use to describe thermalization is the effective kinetic theory (EKT) of Arnold, Moore and Yaffe [26], which is leading order accurate in the QCD coupling constant $\lambda=g^{2} N_{c}=$ $4 \pi \alpha_{s} N_{c}$ in the combined limit of weak coupling $(\lambda \rightarrow 0)$ and nonperturbative occupancies $(\lambda f \rightarrow 0)$ for modes whose momenta are larger than the thermal screening scale in the nonequilibrium system $p^{2} \gg m^{2} \sim \lambda \int d^{3} \mathbf{p} f(\mathbf{p}) / p$.

At leading order in the coupling constant, the EKT describes the time evolution of color- or spin-averaged distribution function $f_{s}$ with an effective $2 \leftrightarrow 2$ scattering and a $1 \leftrightarrow 2$ effective splitting terms. The resulting Boltzmann equation for a homogeneous nonexpanding system is

$$
\partial_{t} f_{s}(\mathbf{p}, t)=-\mathcal{C}_{2 \leftrightarrow 2}^{s}[f](\mathbf{p})-\mathcal{C}_{1 \leftrightarrow 2}^{s}[f](\mathbf{p})
$$

with massless dispersion relation $p^{0}=|\mathbf{p}|=p$. The index $s$ refers to different particle species in the theory. Expanding upon previous implementations of pure gauge theories in Refs. [6,9,10,33] to QCD, $s$ now stands for gluons and $2 N_{f}$ massless fermions (with quarks and antiquarks counted separately). ${ }^{2}$ The symmetrized $2 \leftrightarrow 2$ collision terms in the right-hand side of Eq. (1) reads

$$
\begin{aligned}
\mathcal{C}_{2 \leftrightarrow 2}^{s}[f](\tilde{\mathbf{p}})= & \frac{1}{2} \frac{1}{\nu_{s}} \frac{1}{4} \sum_{a b c d} \int_{\mathbf{p k p}^{\prime} \mathbf{k}^{\prime}}\left|\mathcal{M}_{c d}^{a b}\right|^{2}(2 \pi)^{4} \delta^{(4)}\left(p^{\mu}+k^{\mu}-p^{\prime \mu}-k^{\prime \mu}\right) \\
& \times\left\{\left(f_{\mathbf{p}}^{a} f_{\mathbf{k}}^{b}\left(1 \pm f_{\mathbf{p}^{\prime}}^{c}\right)\left(1 \pm f_{\mathbf{k}^{\prime}}^{d}\right)\right)-\left(f_{\mathbf{p}^{\prime}}^{c} f_{\mathbf{k}^{\prime}}^{d}\left(1 \pm f_{\mathbf{p}}^{a}\right)\left(1 \pm f_{\mathbf{k}}^{b}\right)\right)\right\} \\
& \times(2 \pi)^{3}\left[\delta^{(3)}(\tilde{\mathbf{p}}-\mathbf{p}) \delta_{a s}+\delta^{(3)}(\tilde{\mathbf{p}}-\mathbf{k}) \delta_{b s}-\delta^{(3)}\left(\tilde{\mathbf{p}}-\mathbf{p}^{\prime}\right) \delta_{c s}-\delta^{(3)}\left(\tilde{\mathbf{p}}-\mathbf{k}^{\prime}\right) \delta_{d s}\right],
\end{aligned}
$$

where $\left|\mathcal{M}_{c d}^{a b}\right|^{2}$ is a $2 \leftrightarrow 2$ scattering amplitude squared summed over all d.o.f. of the external legs $\left[\nu_{q}=2 N_{c}\right.$ for quarks and $\nu_{g}=2\left(N_{c}^{2}-1\right)$ for gluons], $\sum_{a b c d}$ is a sum over all particle and antiparticle species, and $\int_{\mathbf{p}}=\frac{d^{3} \mathbf{p}}{2 p(2 \pi)^{3}}$ is a shorthand notation for Lorentz-invariant momentum integral. The second line is the usual phase-space loss and gain terms, while the Kronecker and Dirac delta functions in the last line accounts for the possibility of particle $s$ to be on any of the four external lines. Finally the numerical prefactors in front of the integral correct the double counting of identical processes.

\footnotetext{
${ }^{1}$ We note that there are also other nonperturbative (in the sense that they involve an infinite number of diagrams) but weak-coupling (in the sense that they can be expanded in a series in the coupling constant) processes which do not contribute to our leading order calculation. These processes include for example the sphaleron transitions; see [32].

${ }^{2}$ In this work we consider plasma at a zero chemical potential with quark and antiquark distributions being equal.
} 
The effective matrix elements $\left|\mathcal{M}_{c d}^{a b}\right|^{2}$ in Eq. (2) are for most kinematics the normal tree-level vacuum matrix element (see Table II in Ref. [26]). For soft small angle scatterings with energy transfer $\omega \ll p, k$, the tree-level Coulomb and Compton scatterings are infrared divergent, elevating a set of diagrams with an arbitrary number of loops to the same magnitude as the tree-level diagrams. These effects become important at the in-medium screening scale $p \sim m_{g}, m_{q}$. Here the in-medium effective masses of gluon and quarks are given, respectively, by

$$
m_{g}^{2}=2 g^{2} \int_{\mathbf{p}}\left[2 C_{A} f_{g}(\mathbf{p})+2 N_{f} C_{F} \frac{\nu_{q}}{\nu_{g}}\left(f_{q}(\mathbf{p})+f_{\bar{q}}(\mathbf{p})\right)\right],
$$

$$
m_{q}^{2}=2 g^{2} \int_{\mathbf{p}}\left[2 C_{F} f_{g}(\mathbf{p})+C_{F}\left(f_{q}(\mathbf{p})+f_{\bar{q}}(\mathbf{p})\right)\right] .
$$

For momentum transfer of this order the dispersion of the internal line in the computation of $\left|\mathcal{M}_{c d}^{a b}\right|^{2}$ gets an $\mathcal{O}(1)$ correction from the in-medium physics. Therefore, for the problematic soft scattering we replace the matrix element with that computed in the hard thermal loop (HTL) approximation that self-consistently treats the medium interaction correctly to leading order. We perform this substitution by removing the infrared divergent small angle approximation from the full matrix element and replace it with the small angle approximation of the full HTL rate [37]. Specifically for a soft gluon or fermion exchange with the momentum transfer $q=\left|\mathbf{p}^{\prime}-\mathbf{p}\right|$ in the $t$ channel, the divergent term $(u-s) / t \sim 1 / q^{2}$ is replaced by the IR regulated term

$$
\frac{u-s}{t} \rightarrow \frac{u-s}{t} \frac{q^{2}}{q^{2}+\xi_{s}^{2} m_{s}^{2}}
$$

where $\xi_{g}=e^{5 / 6} / 2$ and $\xi_{q}=e / 2$ are fixed such that the matrix element reproduces the full HTL results for drag and momentum diffusion properties of soft gluon scattering [37] and gluon to quark conversion $g g \rightarrow q \bar{q}[38,39]$ at leading order for isotropic distributions.

While the soft $\omega \sim m_{g}$ scatterings do not appreciably change the momentum state of the particle, they may bring the particle slightly off shell and make it kinematically possible for the particle to decay through nearly collinear splitting. This makes the effective $1 \leftrightarrow 2$ matrix element a leading order effect. It is included as $\mathcal{C}^{1 \leftrightarrow 2}[f](\tilde{\mathbf{p}})$ on the right-hand side of the Boltzmann equation (1) and explicitly

$$
\begin{aligned}
& \mathcal{C}_{1 \leftrightarrow 2}^{s}[f](\tilde{\mathbf{p}}) \\
& =\frac{1}{2} \frac{1}{\nu_{s}} \sum_{a b c} \int_{0}^{\infty} d p d p^{\prime} d k^{\prime} 4 \pi \gamma_{b c}^{a}\left(p ; p^{\prime}, k^{\prime}\right) \delta\left(p-p^{\prime}-k^{\prime}\right) \\
& \quad \times\left\{f_{p \hat{\mathbf{n}}}^{a}\left[1 \pm f_{p^{\prime} \mathbf{n}}^{b}\right]\left[1 \pm f_{k^{\prime} \mathbf{n}}^{c}\right]-f_{p^{\prime} \hat{\mathbf{n}}}^{b} f_{k^{\prime} \mathbf{\mathbf { n }}}^{c}\left[1 \pm f_{p \hat{\mathbf{n}}}^{a}\right]\right\} \\
& \quad \times \frac{(2 \pi)^{3}}{4 \pi \tilde{p}^{2}}\left[\delta(\tilde{p}-p) \delta_{a s}-\delta\left(\tilde{p}-p^{\prime}\right) \delta_{b s}-\delta\left(\tilde{p}-k^{\prime}\right) \delta_{c s}\right],
\end{aligned}
$$

where the unit vector $\hat{\mathbf{n}}=\tilde{\mathbf{p}} /|\tilde{\mathbf{p}}|$ defines the splitting direction and $\gamma_{b c}^{a}\left(p ; p^{\prime}, k^{\prime}\right)$ is the effective collinear splitting rate including Landau-Pomeranchuk-Migdal [28-31] suppression of collinear radiation. Factoring out the kinematic splitting functions, the rates

$$
\begin{gathered}
\gamma_{g g}^{g}\left(p ; p^{\prime}, k^{\prime}\right)=\frac{p^{4}+p^{\prime 4}+k^{\prime 4}}{p^{3} p^{\prime 3} k^{\prime 3}} \mathcal{F}_{g}\left(p ; p^{\prime}, k^{\prime}\right), \\
\gamma_{q g}^{q}\left(p ; p^{\prime}, k^{\prime}\right)=\frac{p^{2}+p^{\prime 2}}{p^{2} p^{\prime 2} k^{\prime 3}} \mathcal{F}_{q}\left(p ; p^{\prime}, k^{\prime}\right), \\
\gamma_{q \bar{q}}^{g}\left(p ; p^{\prime}, k^{\prime}\right)=\gamma_{q g}^{q}\left(k^{\prime} ;-p^{\prime}, p\right)
\end{gathered}
$$

are given by an effective vertex resuming an infinite number of possible soft interactions with the medium [26]. It is found by solving the following integral equation:

$$
\begin{aligned}
2 \mathbf{h}= & i \delta E(\mathbf{h}) \mathbf{F}_{s}(\mathbf{h})+g^{2} T_{*} \int \frac{d^{2} \mathbf{q}_{\perp}}{(2 \pi)^{2}} \mathcal{A}\left(\mathbf{q}_{\perp}\right) \\
& \times\left\{\frac{1}{2}\left(C_{s}+C_{s}-C_{A}\right)\left[\mathbf{F}_{s}(\mathbf{h})-\mathbf{F}_{s}\left(\mathbf{h}-k^{\prime} \mathbf{q}_{\perp}\right)\right]\right. \\
& +\frac{1}{2}\left(C_{s}+C_{A}-C_{s}\right)\left[\mathbf{F}_{s}(\mathbf{h})-\mathbf{F}_{s}\left(\mathbf{h}-p^{\prime} \mathbf{q}_{\perp}\right)\right] \\
& \left.+\frac{1}{2}\left(C_{A}+C_{s}-C_{s}\right)\left[\mathbf{F}_{s}(\mathbf{h})-\mathbf{F}_{s}\left(\mathbf{h}+p \mathbf{q}_{\perp}\right)\right]\right\},
\end{aligned}
$$

and $\mathcal{F}_{s}\left(p ; p^{\prime}, k^{\prime}\right)$ is defined as

$\mathcal{F}_{s}\left(p ; p^{\prime}, k^{\prime}\right)=\frac{\nu_{s} C_{s} g^{2}}{8(2 \pi)^{4}} \int \frac{d^{2} h}{(2 \pi)^{2}} 2 \mathbf{h} \cdot \operatorname{Re} \mathbf{F}_{s}\left(\mathbf{h} ; p, p^{\prime}, k^{\prime}\right)$.

In this work the strength of soft momentum background fluctuations $\mathcal{A}\left(\mathbf{q}_{\perp}\right)$ is treated using an isotropic screening approximation [40]:

$$
\mathcal{A}\left(\mathbf{q}_{\perp}\right)=\frac{1}{\mathbf{q}_{\perp}^{2}}-\frac{1}{\mathbf{q}_{\perp}^{2}+2 m_{g}^{2}},
$$

the energy difference $\delta E$ is defined as 


$$
\delta E\left(\mathbf{h} ; p, p^{\prime}, k^{\prime}\right) \equiv \frac{m_{g}^{2}}{2 k^{\prime}}+\frac{m_{s}^{2}}{2 p^{\prime}}-\frac{m_{s}^{2}}{2 p}+\frac{\mathbf{h}^{2}}{2 p k^{\prime} p^{\prime}},
$$

and the effective temperature $T_{*}$ is given by

$T_{*} \equiv \frac{1}{\nu_{g} m_{g}^{2}} \sum_{s} \nu_{s} g^{2} C_{s} \int \frac{d^{3} p}{(2 \pi)^{3}} f_{s}(\mathbf{p})\left(1 \pm f_{s}(\mathbf{p})\right)$.

Instead of solving Eq. (10) directly, the required twodimensional integral Eq. (11) is expressed as the value at the origin of the Fourier transformed function $\tilde{\mathbf{F}}_{s}$, which solves a Fourier transformed Eq. (10). We solve it using the basis function method [41] and parametrize the solution for the Monte Carlo sampling of the collision kernel in Eq. (6). For the distribution functions we use a previously developed discretization scheme that does not introduce additional discretization errors for energy or particle number densities [6,10,37]. The distribution functions are discretized in spherical polar coordinates on a logarithmic momentum grid with typical momentum range $p_{\max } / p_{\min }=1500$ and $N_{p}=100$. For anisotropically expanding systems the longitudinal momentum fraction $\cos \theta=p_{z} / p$ is discretized on a uniform grid with a typical value of $N_{\theta}=200$. We considered azimuthally symmetric distributions. The collision integrals were calculated by Monte Carlo sampling of the phase space with importance sampling [6]. At each time step the $2 \leftrightarrow 2$ collision integral Eq. (2) was calculated using $N_{2 \leftrightarrow 2} N_{p} N_{\theta}$ randomly generated vector quadruplets $\mathbf{p}, \mathbf{k}, \mathbf{p}^{\prime}, \mathbf{k}^{\prime}$ satisfying the momentum and energy conservation, where $N_{2 \leftrightarrow 2}=50$, 100. For $1 \leftrightarrow 2$ collision integral Eq. (6) we used $N_{1 \leftrightarrow 2} N_{p}$ samples of momentum $p, p^{\prime}, k^{\prime}=p-p^{\prime}$ combinations, which were reused for each angular direction (here $\left.N_{1 \leftrightarrow 2}=50,100\right)$.

\section{CHEMICAL EQUILIBRATION IN ISOTROPIC NONEXPANDING SYSTEMS}

\section{A. Kinetically equilibrated initial conditions}

In order to gain intuition to the far-from-equilibrium dynamics, we start with a particularly simple system where gauge bosons and fermions are initialized at time $t=0$ with thermal distributions

$$
f_{\mathrm{eq}}^{s}(p)=\frac{1}{e^{p / T_{s, \text { init }}} \pm 1},
$$

but with different initial temperatures $T_{\mathrm{g} \text {,init }} \neq T_{\mathrm{q} \text {,init }}$. In such a situation we say that quarks and gluons are in kinetic equilibrium among themselves but not in thermal equilibrium with each other. The system will relax into a state in which both the quarks and the gluons are in equilibrium with the same temperature $T_{\text {final }}$. The energy conservation dictates that the final temperature will be given by $\nu_{g} T_{g, \text { init }}^{4}+2 N_{f} \nu_{q} \frac{7}{8} T_{q, \text { init }}^{4}=\left(\nu_{g}+2 N_{f} \nu_{q} \frac{7}{8}\right) T_{\text {final }}^{4}$.

If we start with a pure gluon initial state, i.e., $T_{q \text {,init }}=0$, the fermion number is initially zero. In order to reach chemical equilibrium, the fermion number has to be subsequently generated by pair production either through medium-induced $g \rightarrow q \bar{q}$-splitting processes or alternatively through $g g \rightarrow q \bar{q}$ conversions. Multiplying the Boltzmann equation (1) by $2 N_{f} \nu_{q} p^{2} / \lambda^{2} T^{3}$ for quarks, we obtain the equation for the rate of change of fermion number (per momentum)

$$
\frac{2 N_{f} \nu_{q}}{\lambda^{2} T^{3}} \partial_{t}\left[p^{2} f_{q}(\mathbf{p}, t)\right]=C_{22}^{q}+C_{12}^{q}
$$

and similarly for gluons. In Fig. 1 we show the rates $C_{22}^{s}$ and $C_{12}^{s}$ separately and as a sum for coupling constant $\lambda=0.1$ and temperature $T=T_{g \text {,init }}$. We see that the $2 \leftrightarrow 2$ processes dominate fermion production around $p \sim T$ (blue dashed line), while for $p \sim m_{D}=\sqrt{2} m_{g}$ the splitting processes become roughly equally important (blue dotted line). The changes in the gluon distribution mirror the fermionic ones. We see that $g g \rightarrow q \bar{q}$ conversion reduces the number of gluons at the same momentum scale $p \sim T$ where fermions are created (blue and red dashed lines), while soft collinear radiation from $p \sim T$ gluons (red dotted line) produces soft fermions. Importantly, the resulting fermion spectrum is nonthermal.

We now turn to study how the above system evolves toward equilibrium as a function of time. For the same $\lambda=0.1$ as above, Fig. 2 displays the time evolution of several different effective temperatures $T_{g / q, \alpha}^{\mathrm{eff}}$, defined

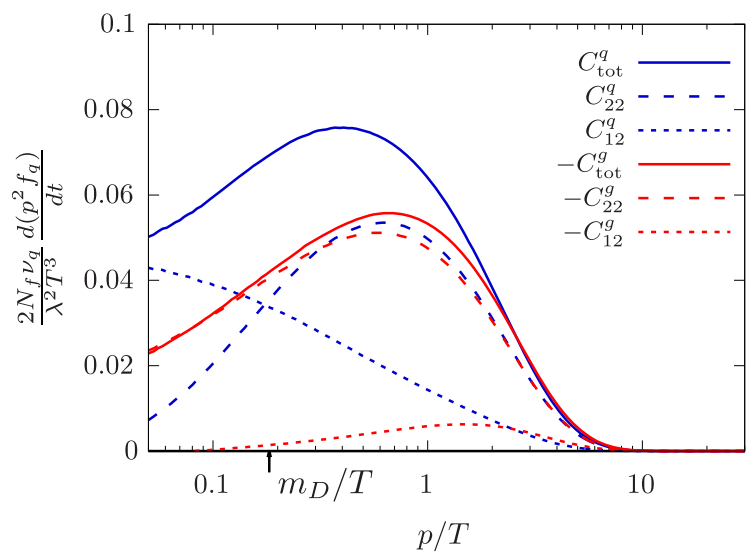

FIG. 1. The rate of change of total fermion number density (blue lines) for a thermal gluon system with no initial quark density and $\lambda=0.1$. The dashed and dotted lines show the contributions to the rate from elastic $2 \leftrightarrow 2$ and inelastic $1 \leftrightarrow 2$ processes, while the solid lines are the sum of the two. The corresponding changes in the gluon number density (multiplied by -1 ) are shown by red lines. 


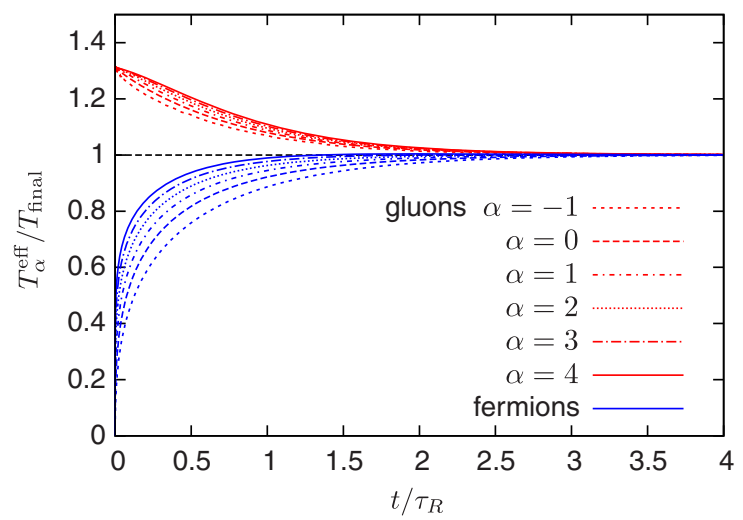

FIG. 2. The various effective temperatures [see Eq. (18)] as a function of time for a nonexpanding system with initial conditions of thermal gluons and no fermions. The time axis is scaled by $\tau_{R}=4 \pi \eta / s / T_{\text {final }}$, where $\eta / s \approx 1900$ for $\lambda=0.1$. The red lines are the gluonic effective temperatures; the blue lines are the effective temperatures of the fermionic sector with $T_{4}^{\mathrm{eff}}>T_{3}^{\mathrm{eff}}>\cdots>T_{-1}^{\mathrm{eff}}$.

through the $\alpha$ th moments of the fermion $(F=1)$ and boson $(F=0)$ distribution functions

$$
T_{g / q, \alpha}^{\mathrm{eff}}=\left[\mathcal{N}_{\alpha, F} \int \frac{d^{3} p}{(2 \pi)^{3}} p^{\alpha} f(p)\right]^{\frac{1}{\alpha+3}} .
$$

The effective temperatures are normalized such that when the system is in thermal equilibrium all $T_{g / q, \alpha}^{\mathrm{eff}}($ for all $\alpha$ ) are equal to the equilibrium temperature $T_{g / q, \alpha}^{\mathrm{eff}}=T_{\text {final }}$, that is,

$$
\mathcal{N}_{\alpha, F}=\left[1-2^{-\alpha-2}\right]^{-F} \frac{2 \pi^{2}}{\Gamma(\alpha+3) \zeta(\alpha+3)} .
$$

Lower $\alpha$ values are more sensitive to the infrared of the distribution, whereas larger $\alpha$ values describe the UV part of the distribution function. In particular, $T_{1}^{\text {eff }} \propto \sqrt[4]{e}$ corresponds to the temperature of a fictitious thermally equilibrated system with the same energy density.

We display the effective temperatures as a function of relaxation time of the final thermalized system

$$
\tau_{R}=\frac{4 \pi \eta / s}{T_{\text {final }}},
$$

where $\eta / s$ is the specific shear viscosity, whose relation to $\lambda$ is discussed in the Appendix. This relaxation time is parametrically of order of the transport mean free path of the thermalized system $\tau_{R} \sim\left(\lambda^{2} T_{\text {final }}\right)^{-1}$, but it has been observed in $[6,10,42,43]$ that expressing the coupling constant $\lambda$ in terms of the specific viscosity accounts for large numerical corrections that go beyond the parametric expression and leads to better scaling behavior for different values of $\lambda$.
We first note that the effective temperatures of gluons (red lines in Fig. 2) decrease while those of the quarks (blue lines) increase. During this evolution the gluon effective temperatures approximately overlap, signifying that gluons remain close to the kinetic equilibrium through the whole evolution. In contrast to gluons-and consistent with the nonequilibrium spectrum of the fermion production rate in Fig. 1-the fermion effective temperatures differ until full chemical equilibration is achieved. During this evolution the fermion spectrum is harder than that in kinetic equilibrium as seen from the ordering of the effective temperatures $T_{4}^{\text {eff }}>T_{3}^{\text {eff }}>\cdots>T_{-1}^{\text {eff }}$.

In chemical equilibrium $N_{f}=3$ fermions constitute $e_{q, \text { eq }} / e_{q, \text { total }} \approx 0.66$ of the total equilibrium energy density [see Eq. (16)]. It can be seen in Fig. 3 that by the time $t_{\text {chem }} \approx 1.4 \tau_{R}$ for $\lambda=0.1$ the fermion energy density $e_{q}$ has reached $90 \%$ of its equilibrium value $e_{q \text {,final }}$, which we take as our somewhat arbitrary definition of the chemical equilibration time, i.e.,

$$
\frac{e_{q}\left(t_{\mathrm{chem}}\right)}{e_{q, \text { final }}}=\left(\frac{T_{1, q}^{\mathrm{eff}}\left(t_{\mathrm{chem}}\right)}{T_{\text {final }}}\right)^{4}=0.9 .
$$

To study the coupling dependence of the chemical equilibration, we repeat the above calculation with the same initial conditions but with several different values of $\lambda=10,1.0,0.1$, corresponding to $\eta / s \approx 1,35,1900$, respectively. Figure 3 shows the time evolution of fermion energy fraction for these different values of 't Hooft couplings, for which the chemical equilibration time varies by 3 orders of magnitude. However, as is seen from the figure, the functional forms of the time evolutions of the energy densities follow closely a common form when described in terms of the relaxation time $\tau_{R}$, with the system reaching chemical equilibrium around

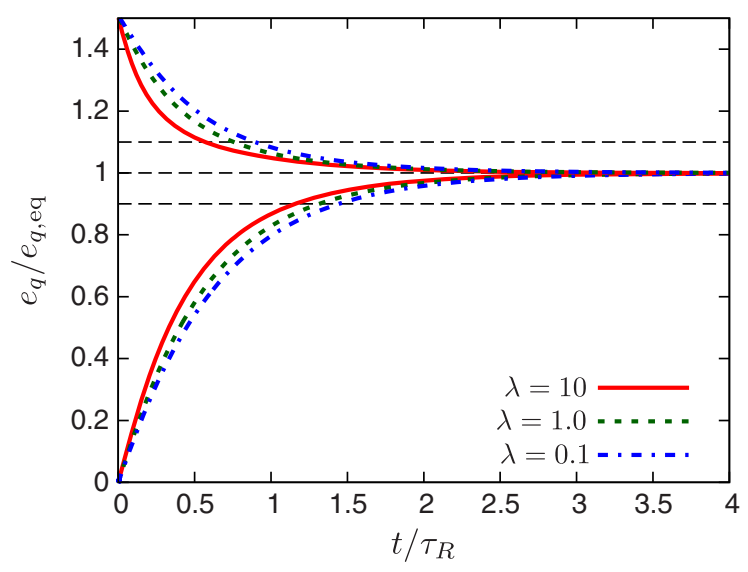

FIG. 3. Chemical equilibration of fermion energy density (as a function of equilibrium energy density) for different coupling constants $\lambda=10,1.0,0.1$, which correspond to effective $\eta / s=1,35,1900$. Two cases shown: with zero initial fermion energy $e_{q} \approx 0$ and with zero initial gluon energy $e_{g} \approx 0$. 


$$
t_{\text {chem }} / \tau_{R} \sim 1.1-1.4
$$

for all studied values of $\lambda$.

We note however that for moderate values $\lambda \gtrsim 10$, we expect substantial next-to-leading order corrections to transport properties of QGP [44]. Nevertheless, while the kinetic theory suffers from large systematic uncertainties for these values of $\lambda$, the fact that the kinetic theory calculation itself does not fail catastrophically may allow one to extrapolate the results for semiquantitative order of magnitude estimates even at larger values of $\lambda$ using $\tau_{R}$ scaling, where the dependence on the coupling constant only enters through the specific shear viscosity $\eta / s$. Note that other transport coefficients in units of $\eta / s$, e.g., $\tau_{\pi} /(\eta /(s T))$, are much less sensitive to the changes of the coupling constant [45].

We note in passing that similar qualitative features of the equilibration are seen by varying the initial starting gluon and fermion temperatures. In particular, the gluons remain in approximate kinetic equilibrium throughout evolution even if one starts with fermion-dominated initial conditions (data not shown). In this case the fermion energy fraction approaches equilibrium ratio from above (see Fig. 3) and the chemical equilibration takes place at $t_{\text {chem }} / \tau_{R} \sim$ $0.5-0.9$, where now $e_{q}\left(t_{\text {chem }}\right) / e_{q, \text { final }}=1.1$ in this case.

\section{B. Overoccupied initial conditions}

We now turn to a system starting with an overoccupied distribution of gluons and no fermions. This system has initially too many gluons, $f_{g} \gg 1$, with too soft momenta compared to a thermal ensemble with the same energy density, that is, $T_{2}^{\text {eff }} \ll T_{1}^{\text {eff }} \ll T_{0}^{\text {eff }}$. As the fermions cannot be overoccupied, such a system is necessarily dominated by the gluons in the early stages. As discussed in detail in [9,37,46-51], overoccupied gluonic systems thermalize via a self-similar cascade which carries the energy and particle number from the infrared to the ultraviolet via elastic and inelastic scattering. As long as the system is parametrically overoccupied $f_{g} \gg 1$, the cascade is self-similar; that is, the gluon distribution function at a given time $t$ is given by an approximately stationary scaling function $\tilde{f}$ which is insensitive to the initial conditions

$f_{g}(p)=(Q t)^{-4 / 7} \tilde{f}\left(p / p_{\max }\right), \quad p_{\max }=Q(Q t)^{1 / 7}$,

where $Q^{4}=2 \pi^{2} \lambda \int_{\mathbf{p}} p f_{g}(p)$. This scaling form is reached in a time that is proportional to the scattering rate of the initial condition [9], which is parametrically faster than the thermalization time for a parametrically overoccupied system. The approach to the scaling form is discussed in detail in [51]. Once the typical momentum of the cascade $p_{\max }$ reaches the thermal scale $T_{\text {final }}$, the system equilibrates. How the presence of fermions may affect the cascade has been studied using (semi)classical Yang-Mills

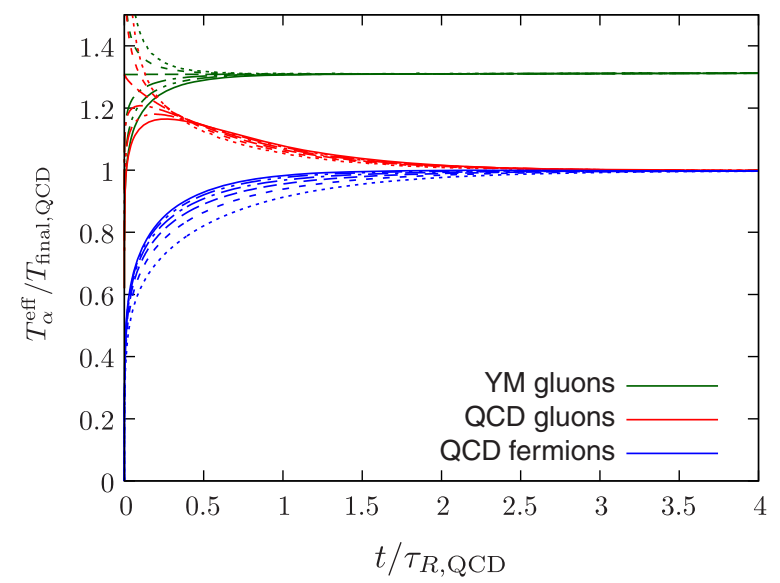

FIG. 4. Chemical equilibration for an isotropic overoccupied gluon state with no initial fermions present. The effective temperature $T_{\alpha}$ of gluon (red lines) and fermion (blue lines) distribution functions are shown for $\lambda=0.1$. Note that for fermions $T_{4}^{\text {eff }}>T_{3}^{\text {eff }}>\cdots>T_{-1}^{\text {eff }}$, but overoccupied gluons start with inverted ordering.

simulations in the regime where the gluons are still highly overoccupied and $p_{\max } \ll T_{\text {final }}$ [52]. We now answer the question of what happens when $p_{\max } \sim T$.

The green lines in Fig. 4 show the time evolution of a pure $N_{c}=3$ gauge theory with overoccupied gluon initial conditions. ${ }^{3}$ For the effective temperatures, this scaling form Eq. (22) corresponds to a power-law behavior

$$
T_{\alpha}^{\mathrm{eff}} \propto t^{\frac{1}{7} \alpha-1}
$$

If the system is parametrically overoccupied, the effective temperatures are also parametrically separated $T_{\alpha}^{\mathrm{eff}} \gg T_{\alpha+1}^{\mathrm{eff}}$, but when the system thermalizes around $t \approx 0.3 \tau_{R}$, the effective temperatures again collapse. Figure 4 also shows the time evolution of a QCD plasma $\left(N_{c}=3, N_{f}=3\right)$ with the same initial condition of overoccupied gluons with no quarks present. The time evolution differs now from the pure glue system as the fermion number is dynamically generated. At early times the quark effective temperatures are small and gluonic temperatures approximately follow the classical power laws. The cascade ends and the gluonic sector reaches a kinetic equilibrium among themselves again around

$$
t \sim t_{\text {kinetic }}^{g} \approx 0.2 \tau_{R},
$$

where we have defined the kinetic equilibration timein analogy with the chemical equilibration time-by demanding

\footnotetext{
${ }^{3}$ The initial condition of the simulation is given by Eq. (29) with $\lambda=0.1$ and $\xi=1$. However as the system very quickly reaches the self-similar scaling form, the details of the initial condition matter only at very early times $t \ll \tau_{R}$.
} 


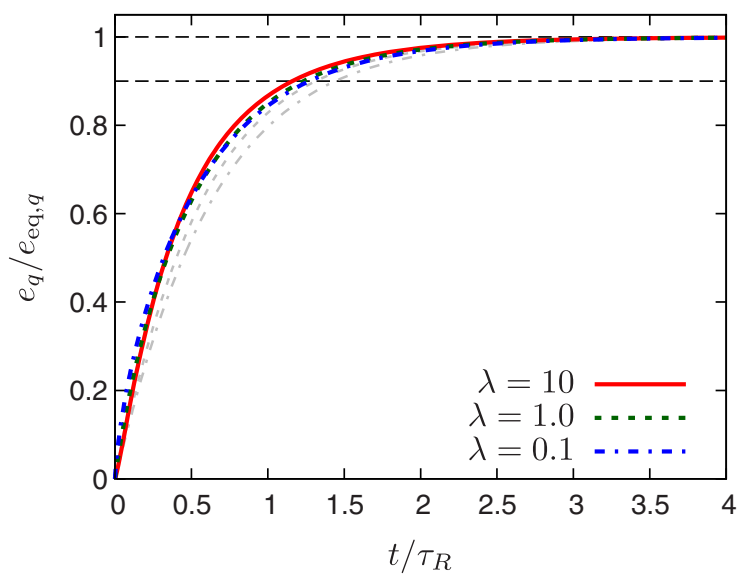

FIG. 5. Chemical equilibration of fermion energy density (as a function of equilibrium energy density) for different coupling constants $\lambda=10,1.0,0.1$, which correspond to effective $\eta / s=1,35,1900$ for the system initialized with overoccupied gluon density (colored lines) and thermal initial conditions (gray lines).

$$
\left(\frac{T_{0}^{g}\left(t_{\text {kinetic }}\right)}{T_{1}^{g}\left(t_{\text {kinetic }}\right)}\right)^{4}=0.9
$$

This timescale is significantly faster than the timescale for chemical equilibration $t_{\text {chem }}$. Indeed at the time the kinetic equilibrium among gluons is reached, there are only a few quarks present and the state of the system is approximately the same as in the case of thermal initial conditions studied in the previous section. From this point on, the chemical equilibrium follows the same pattern which was described in the previous section. To emphasize this point, in Fig. 5 we show the fermion energy fraction for overoccupied initial conditions (colored lines) on top of the thermal initial conditions (gray lines), which were shown in Fig. 3.

\section{CHEMICAL EQUILIBRATION IN EXPANDING SYSTEMS}

In the weak-coupling description of heavy-ion collisions the state of the system right after the initial particle production is given by an overoccupied distribution of gluons [35,36]. The novel feature compared to the previous section is that the geometry of the collision system is such that the overoccupied matter is undergoing a rapid, approximately boost-invariant longitudinal expansion. Such a system has been studied in detail in pure gauge theory $[10,33]$ and it forms the link between the initial condition and the hydrodynamic stage in phenomenological multistage simulations of heavy-ion collisions $[42,43]$. Here, we study the expanding plasma in full QCD.

Assuming a boost-invariant form of the distribution function, the Boltzmann equation can be written in the form [18] $\partial_{\tau} f_{s}(\mathbf{p}, \tau)-\frac{p^{z}}{\tau} \partial_{p^{z}} f_{s}(\mathbf{p})=-\mathcal{C}_{2 \leftrightarrow 2}^{s}[f](\mathbf{p})-\mathcal{C}_{1 \leftrightarrow 2}^{s}[f](\mathbf{p})$,

where $\tau$ is the Bjorken time $\tau=\sqrt{t^{2}-z^{2}}$. The expansion redshifts the distribution in the $p^{z}$ direction, making it more anisotropic along the longitudinal momentum, while $2 \leftrightarrow 2$ scatterings act to isotropize the system [53]. While anisotropic systems could suffer from the presence of unstable plasma modes [54-56] affecting the kinetic dynamics [50,57], the detailed 3+1D classical-statistical YangMills simulations $[49,58]$ found that late time evolution of anisotropic systems is in agreement with kinetic theory expectations neglecting plasma instabilities [53]. In the absence of a general nonequilibrium formulation of QCD kinetic theory [50], we use QCD kinetic theory with isotropic approximations, which remove the unstable modes, to study the equilibration in expanding systems. Note that there are no unstable fermionic modes $[59,60]$.

While the expansion conserves total energy, the local energy density in a given rapidity slice decreases as a function of time. At late times when the system is close to local thermal equilibrium, the time evolution of the temperature is given by ideal hydrodynamics with constant $T(\tau) \tau^{1 / 3}$. As the target temperature to which the out-ofequilibrium system aims to thermalize changes, so does the kinetic relaxation time. In the following we follow the practice of [42] and, for each simulation, we determine the asymptotic value of $\left.T(\tau) \tau^{1 / 3}\right|_{\tau \rightarrow \infty}$, use the ideal hydrodynamics relation to extract what the temperature of the system would have been at earlier times if the full time evolution of the system were described by ideal fluid dynamics

$$
T_{\mathrm{id}}(\tau)=\frac{\left.\left(T(\tau) \tau^{1 / 3}\right)\right|_{\tau \rightarrow \infty}}{\tau^{1 / 3}}
$$

and use that in our definition of time-dependent kinetic relaxation time

$$
\tau_{R}(\tau)=\frac{4 \pi \eta / s}{T_{\mathrm{id}}(\tau)}
$$

We consider an expanding system with an overoccupied initial condition motivated by the color-glass-condensate framework

$$
f^{g}\left(\mathbf{p}, \tau=\tau_{0}\right)=\frac{2 A}{\lambda} \frac{Q_{0}}{\sqrt{p_{\perp}^{2}+p_{z}^{2} \xi^{2}}} e^{-\frac{2^{2}{ }_{\perp}^{2} \xi^{2}}{Q_{0}^{2}}}
$$

where the values of $A, Q_{0}$, and $\xi$ are adjusted to reproduce the mean transverse momentum squared $\left\langle p_{T}^{2}\right\rangle$ and energy density $e\left(\tau_{0}\right)$ at the initial time $\tau_{0}$ extracted from the classical lattice simulations of initial stages of the 


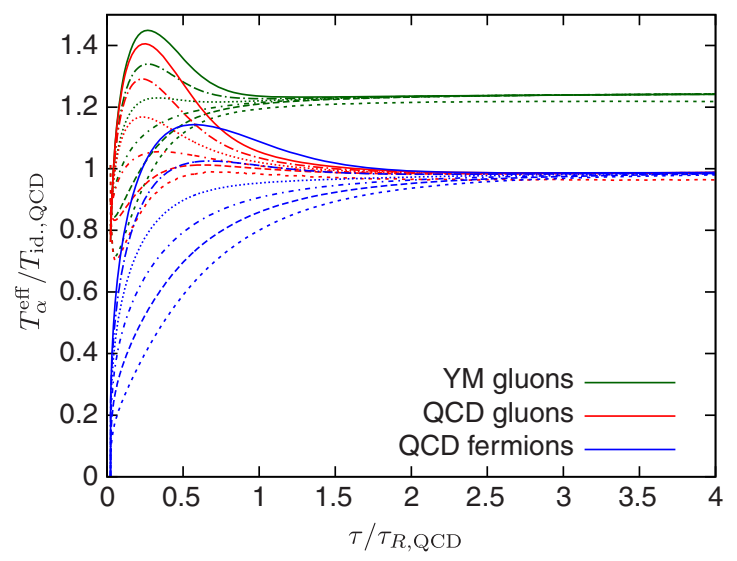

FIG. 6. The effective temperature $T_{\alpha}^{\text {eff }}(\alpha=-1, \ldots, 4)$ evolution in a longitudinally expanding system for QCD $\left(N_{c}=3, N_{f}=3\right)$ and Yang-Mills $\left(N_{c}=3, N_{f}=0\right)$. Initial conditions given by an anisotropic overoccupied gluon state $(\lambda=5, \xi=10, \eta / s \approx 2.75)$ with no initial fermions, Eq. (29). Axes are scaled by timedependent relaxation time and asymptotic temperatures $\tau_{R}(\tau)=$ $(4 \pi \eta / s) / T_{\text {id }}(\tau)$ and $T_{\text {id }}(\tau)$.

collision $[10,61]$. The anisotropy parameter $\xi$ determining the ratio of longitudinal to transverse pressure is chosen such that $P_{L} \ll P_{T}$ and is set in the following $\xi=10$. The same initial conditions have been studied in pure gauge theory in $[10,33,42]$. Here, as in $[10,33,42]$, we use $\tau_{0}=$ $1 / Q_{s}$ and $Q_{0}=1.8 Q_{s}$ and we set $A=5.24$. Here $Q_{s}$ is the saturation scale of color glass condensate and is of order $Q_{s}^{-1} \sim 0.1 \mathrm{fm}$.

In Fig. 6 we show the time evolution of the effective temperatures $T_{\alpha}^{\text {eff }}$ for the initial conditions Eq. (29) and $\lambda=5$, which corresponds to $\eta / s \approx 2.75$. The rapid longitudinal expansion quickly inverts the hierarchy of temperatures from an overoccupied state $f^{g} \gg 1\left(T_{\alpha}^{\text {eff }}>T_{\alpha+1}^{\text {eff }}\right)$ to an underoccupied state $f^{g} \ll 1$, which is well understood as the first stage of the bottom-up thermalization [53]. This transition takes place well before a substantial number of fermions are produced as can be seen from the good overlap of the time evolution of the effective temperatures in pure gauge theory with the full theory in Fig. 6. This suggests that during this early phase the presence of fermions does not significantly affect the evolution of bulk quantities. Note that the effective temperatures are sensitive only to the angular averaged distribution function, but because of the longitudinal expansion the system is highly anisotropic.

Next we look at the chemical equilibration of fermion energy fraction of the equilibrium energy density. In Fig. 7 we show the approach to chemical equilibrium for different values of the coupling constant $\lambda$ and an overoccupied initial state. Using the same $a d$ hoc criterion of chemical equilibration as in nonexpanding system Eq. (20), we find that chemical equilibration happens for $\tau_{\text {chem }} \sim 1-2 \tau_{R}$ for a wide range of coupling constants $0.5 \leq \lambda \leq 20$ and only for very small coupling $\lambda=0.1$, we get $\tau_{\text {chem }} \approx 2.8 \tau_{R}$.

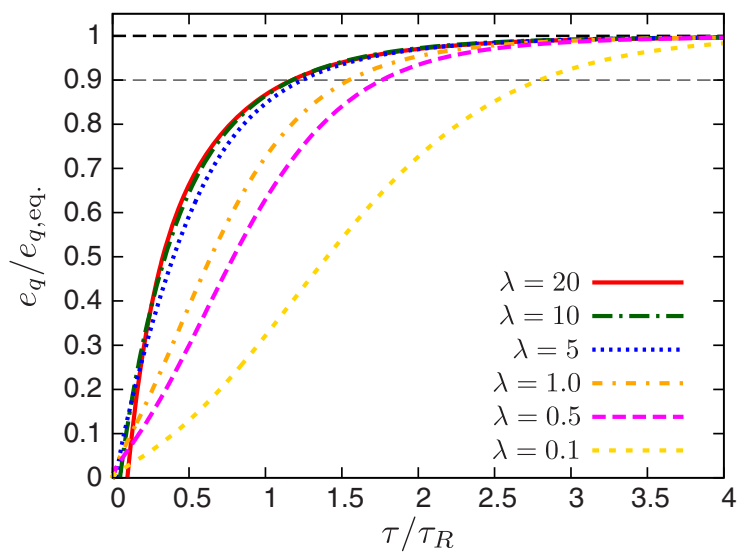

FIG. 7. Fermion energy fraction in a longitudinally expanding system with different coupling constants $\lambda$. The system is initialized with an anisotropic overoccupied gluon state, Eq. (29) $(\xi=10)$. The time axis is scaled by corresponding kinetic relaxation time $\tau_{R}(\tau)$, Eq. (28), for each value of $\lambda$.

We would like to remind that parametrically the chemical equilibration scales as $\sim\left(\lambda^{2} T\right)^{-1}$, so in physical units the equilibration times change by several orders of magnitude. Scaling with relaxation time Eq. (28) reduces this vast separation of scales, and for couplings $\lambda>1$ we observe the collapse of equilibration dynamics to the same curve and the chemical equilibration is reached at $\tau_{\text {chem }} \approx 1.2 \tau_{R}$. The difference in equilibration times for $\lambda \leq 1$, which was not present in the nonexpanding case, arises from the additional scale - the expansion rate $1 / \tau$. Since the starting time $\tau_{0}=1 / Q_{s}$ is kept fixed, for smaller values of the coupling constant, the system experiences a longer phase where the anisotropy is still increasing, i.e., the first stage of bottom up, which delays equilibration.

We note that the chemical equilibration takes place when the system is still highly anisotropic. This is seen in Fig. 8(a) depicting the time evolution of the ratio of the longitudinal and transverse pressures

$$
\begin{gathered}
P_{L}=\int \frac{d^{3} p}{(2 \pi)^{3}} \frac{p_{z}^{2}}{p^{0}}\left(\nu_{g} f_{g}+2 N_{f} \nu_{q} f_{q}\right), \\
P_{T}=\frac{1}{2} \int \frac{d^{3} p}{(2 \pi)^{3}} \frac{p_{x}^{2}+p_{y}^{2}}{p^{0}}\left(\nu_{g} f_{g}+2 N_{f} \nu_{q} f_{q}\right) .
\end{gathered}
$$

The system becomes isotropic only at very late times. At the time of chemical equilibration $\left(\tau \lesssim 3 \tau_{R}\right)$ the pressure anisotropy is still large $P_{L} / P_{T}<0.8$ for all values of $\lambda$. However similarly to the pure gauge theory, the system's time evolution is well described by fluid dynamics well before pressure anisotropies become small; that is, the system exhibits "hydrodynamization without thermalization."

We quantify the approach to thermal equilibrium and hydrodynamization by defining two additional timescales 

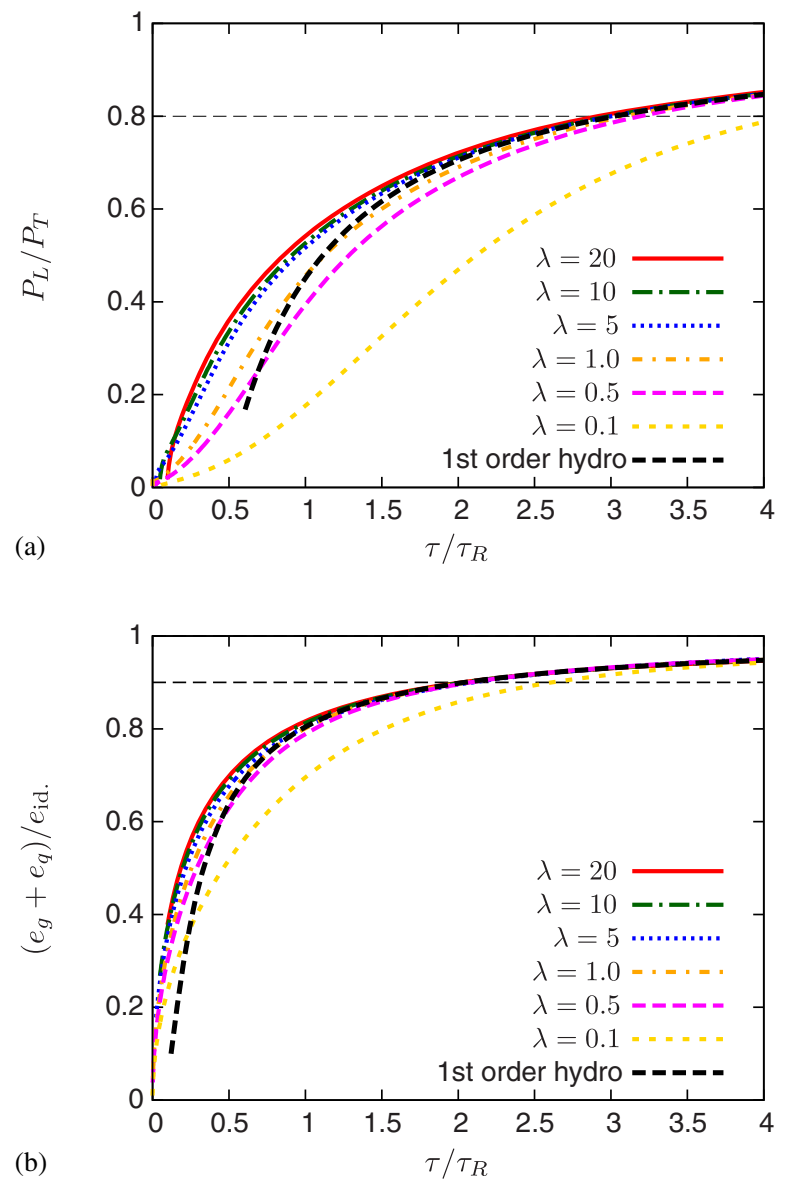

FIG. 8. (a) Total pressure anisotropy $P_{L} / P_{T}$ evolution in kinetic theory with overoccupied initial conditions Eq. (29). (b) Total energy density evolution relative to ideal estimate $e / e_{\mathrm{id}}=\left(T / T_{\mathrm{id}}\right)^{4}$ given by Eq. (27). Comparison with first order viscous temperature Eq. (34) shown by the black dashed curve.

$\tau_{\text {therm }}$ and $\tau_{\text {hydro }}$, in analogy to Eq. (20). We require the combined gluon and fermion energy density $e=e_{g}+e_{q}$ to be within $10 \%$ of ideal and viscous hydrodynamic estimates of energy density

$$
\begin{gathered}
\frac{e\left(\tau_{\text {therm }}\right)}{e_{\text {id }}}=\left(\frac{T\left(\tau_{\text {therm }}\right)}{T_{\text {id }}\left(\tau_{\text {therm }}\right)}\right)^{4}=0.9, \\
\left|1-\frac{e\left(\tau_{\text {hydro }}\right)}{e_{1 \mathrm{st}}}\right|=\left|1-\left(\frac{T\left(\tau_{\text {hydro }}\right)}{T_{1 \mathrm{st}}\left(\tau_{\text {hydro }}\right)}\right)^{4}\right|=0.1 .
\end{gathered}
$$

Here $T_{\text {id }}$ is the ideal estimate of the local temperature Eq. (27) and $T_{1 \text { st }}$ is the first order viscous hydrodynamic solution of longitudinally expanding system with the same late time asymptotics as $T_{\text {id }}$. The analytical solution for first order conformal hydrodynamical equations of motion can be written in units of $\tau_{R}$ as $[62,63]$

$$
\frac{T_{1 \mathrm{st}}(\tau)}{T_{\mathrm{id}}(\tau)}=1-\frac{2}{12 \pi} \frac{\tau_{R}}{\tau}
$$

In Fig. 8(b) we show the time evolution of the total energy density scaled by ideal estimate, i.e., $e / e_{\text {id }}=\left(T / T_{\text {id }}\right)^{4}$. We find that $90 \%$ of equilibrium energy is reached at $\tau_{\text {therm }} \sim$ $2 \tau_{R}$ for the coupling constant values $0.5 \leq \lambda \leq 20$. Only for $\lambda=0.1$ is the approach to equilibrium slower and this criterion is satisfied at $\tau_{\text {therm }} \sim 2.5$. Next, we use the first order hydrodynamic estimate for temperature Eq. (34) and compare it to the kinetic theory. ${ }^{4}$ We achieve agreement with full kinetic theory evolution at very early times and by the time $\tau_{\text {hydro }} \lesssim 0.5 \tau_{R}$ the criterion Eq. (33) is satisfied for $0.5 \leq \lambda \leq 20$. For $\lambda=0.1$ thus defined hydrodynamization takes place somewhat later at $\tau \sim 1.3 \tau_{R}$.

\section{CONCLUSIONS}

In this paper we presented a complete simulation of chemical equilibration in leading order QCD kinetic theory in stationary and expanding systems with infinite transverse extent. By analyzing how out-of-equilibrium plasma of $N_{c}=3$ gluons and $N_{f}=3$ quarks relaxes to the common thermal equilibrium for different values of the coupling constant $\lambda$, we determined the chemical equilibration time in nonexpanding isotropic systems, which we define by requiring the quark energy fraction to be within $10 \%$ of their equilibrium value. For initial conditions with no quarks present, thus defined chemical equilibrium is reached at time $t_{\text {chem }} \sim 1.1-1.4 \tau_{R}$, where $\tau_{R}=(4 \pi \eta / s) / T_{\text {final }}$ is the kinetic relaxation time and $\eta / s(\lambda=10,1.0,0.1) \approx 1,35,1900$. We also note faster gluon dynamics, which results in gauge bosons reaching kinetic equilibrium among themselves before thermalizing with fermions. Consequently, for the case of the overoccupied gluon initial state, gluons first equilibrate through a self-similar cascade as in pure glue theory at times $t_{\text {kinetic }}^{g} \sim 0.2 \tau_{R}$, and then the equilibrium quark densities are produced by the quasithermal gluon background. We would like to emphasise that the chemical equilibration time dependence on the coupling constant $\lambda-$ the only microscopic parameter of the theory-is very well captured by the specific shear viscosity $\eta / s$, which is the macroscopic QGP property. As the relaxation time $\tau_{R}$ changes by orders of magnitude, the equilibration dynamics in rescaled units $t / \tau_{R}$ remains largely unchanged.

Next we studied the QCD equilibration in homogeneous, but longitudinal expanding systems, which is a relevant situation for heavy-ion phenomenology. There the expansion prevents the system from ever reaching static thermal equilibrium and one instead may define thermalization time $\tau_{\text {therm }}$ by requiring the total energy density to be within $10 \%$ of the value given by ideal hydrodynamic evolution $e_{\text {id }}(\tau)=\left(e \tau^{4 / 3}\right)_{\infty} \tau^{-4 / 3}$. Here we note that in the expanding case the effective temperature of the plasma is decreasing

\footnotetext{
${ }^{4}$ Note that substituting Eq. (34) in Eq. (33) generates terms which are formally higher in viscous gradients and could be dropped at first order. We do not do such expansion in Fig. 8 and use the full temperature estimate Eq. (34).
} 
and so we defined time-dependent kinetic relaxation time $\tau_{R}(\tau) \sim \tau^{1 / 3}$ which grows in time; see Eq. (28). For colorglass-condensate-motivated, anisotropic and overoccupied initial conditions, Eq. (29), such thermalization happens at $\tau_{\text {therm }} \sim 2 \tau_{R}(\tau)$ for a range of the coupling constants $0.5 \leq$ $\lambda \leq 20$ but is somewhat delayed for $\lambda=0.1$. The chemical composition of the plasma changes rapidly in the first couple units of relaxation time. Similarly to the nonexpanding systems, gluons undergo a kinetic equilibration faster than fermions, in agreement with two-stage QGP equilibration argued in Ref. [64]. However only when the expansion rate slows down and viscous corrections to the particle distribution function are small enough, particle distributions are well approximated by the equilibrium Bose-Einstein or Fermi-Dirac distributions. Keeping in mind that the effective kinetic relaxation time $\tau_{R}$ is growing in time as the temperature is decreasing, the chemical equilibration in longitudinally expanding systems for moderate values of the coupling constants $\lambda=5,10,20\left(\alpha_{s} \sim 0.1-0.5\right)$ proceeds very similarly in rescaled units to the nonexpanding case and chemical equilibration is reached at $\tau_{\text {chem }} \sim 1.2 \tau_{R}(\tau)$. For smaller values of the coupling constant $\lambda \leq 1$ we do not see the collapse to the same universal curve and the chemical equilibration (in units of $\tau_{R}$ ) takes place later.

In summary, the chemical composition is an important property of the expanding QGP fireball, which is not captured by conventional hydrodynamic modeling of heavy-ion collisions but is essential for the hadrochemistry and photon production, and determines which equation of state best describes the medium. It would be therefore interesting to study if a generalization of hydrodynamics involving nearly conserved charges could be used to describe this nonequilibrium evolution. For realistic values of the coupling constant $\alpha_{s} \sim 0.3$, we find that even in expanding systems the coupling constant dependence can be factored out by rescaling time with kinetic relaxation time $\tau_{R}=(4 \pi \eta / s) / T_{\text {id. }}$, which results in the following ordering of hydrodynamization, chemical equilibration and thermalization timescales

$$
\underbrace{\tau_{\text {hydro }}}_{\lesssim 0.5 \tau_{R}}<\underbrace{\tau_{\text {chem }}}_{\sim 1.2 \tau_{R}}<\underbrace{\tau_{\text {therm }}}_{\sim 2 \tau_{R}}
$$

according to criteria given in Eqs. (20), (33) and (32). Such universality allows one to convert the dimensionless time $\tau / \tau_{R}$ to physical units by matching the late time constants $\left(\tau^{1 / 3} T\right)_{\infty}$ and $\eta / s$ from hydrodynamical modeling of heavy-ion collisions and which is explored in our companion paper [65].

\section{ACKNOWLEDGMENTS}

The authors thank Peter Arnold, Jürgen Berges, Ulrich Heinz, Jacopo Ghiglieri, Jean-François Paquet, Sören Schlichting, Derek Teaney, and Urs Wiedemann for valuable discussions. This work was supported in part by the German Research Foundation (DFG) Collaborative Research Centre (SFB) 1225 (ISOQUANT) (A. M.). Finally, A.M. thanks CERN Theoretical Physics Department for the hospitality during the short-term visit.

\section{APPENDIX: SPECIFIC SHEAR VISCOSITY IN QCD KINETIC THEORY}

The dynamical simulations of the Boltzmann equation with leading order QCD kinetic theory collisions kernels, Eq. (1), allows for the direct determination of the QGP transport properties, for example, the shear viscosity over entropy ratio $\eta / s$. The transport coefficients extracted this way can be compared to calculations of $\eta / s$ using diagonalization of (linearized) collision kernels around thermal equilibrium [7]. In Fig. 9 we show the specific shear viscosity as a function of the coupling constant $\lambda$ obtained from the effective kinetic theory simulations with different, but leading order equivalent IR regulators of the elastic scattering matrix element Eq. (2). The first regulator corresponds to the scheme given by Eq. (5), while in the second case we insert additional $m_{s}^{2} /\left(q^{2}+m_{s}^{2}\right) \sim \mathcal{O}\left(g^{2}\right)$ factors to guarantee the positivity of the scatter matrix element $\left|\mathcal{M}_{c d}^{a b}\right|^{2}$. For $\lambda \lesssim 2$ different implementations of the kinetic theory agree with each other at $\sim 10 \%$. The same level of agreement is also seen with the next-to-leading-log formula, which is a good approximation for the full leading order results [7] (and which corresponds to yet another IR completion of the kinetic theory). For completeness below we summarize the extracted values of $\eta / s$ used in the paper:

\begin{tabular}{lcccccc}
\hline$\lambda$ & 0.1 & 0.5 & 1.0 & 5.0 & 10 & 20 \\
$\eta / s$ & 1900 & 114 & 35 & 2.75 & 1.0 & 0.39 \\
\hline
\end{tabular}

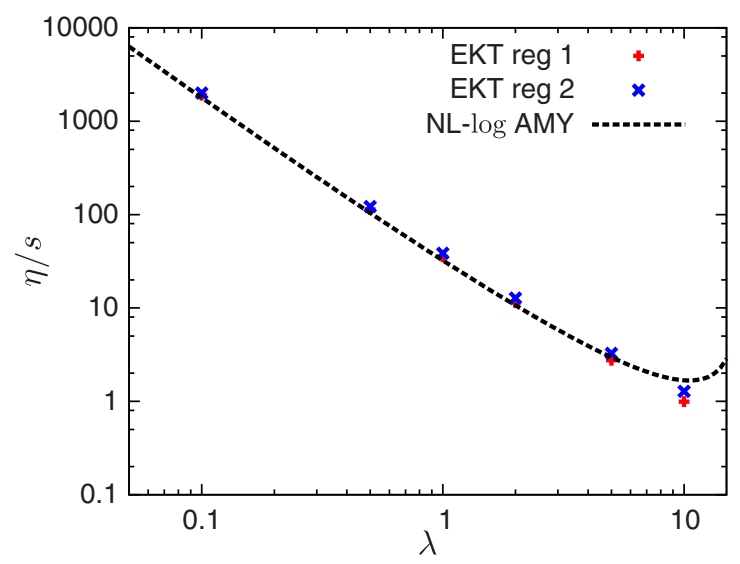

FIG. 9. Shear viscosity over entropy ratio as a function of the coupling constant $\lambda=N_{c} g^{2}$ in our leading order kinetic theory implementation with two regularization schemes of the elastic collision kernel (see the text). The dashed line corresponds to the next-to-leading-log result from Ref. [7]. 
[1] W. Busza, K. Rajagopal, and W. van der Schee, Heavy ion collisions: The big picture, and the big questions, Annu. Rev. Nucl. Part. Sci. 68, 339 (2018).

[2] H.-T. Ding, F. Karsch, and S. Mukherjee, Thermodynamics of strong-interaction matter from lattice QCD, Int. J. Mod. Phys. E 24, 1530007 (2015).

[3] M. P. Heller, R. A. Janik, and P. Witaszczyk, The Characteristics of Thermalization of Boost-Invariant Plasma from Holography, Phys. Rev. Lett. 108, 201602 (2012).

[4] M. P. Heller and M. Spalinski, Hydrodynamics Beyond the Gradient Expansion: Resurgence and Resummation, Phys. Rev. Lett. 115, 072501 (2015).

[5] M. P. Heller, A. Kurkela, M. Spaliski, and V. Svensson, Hydrodynamization in kinetic theory: Transient modes and the gradient expansion, Phys. Rev. D 97, 091503 (2018).

[6] L. Keegan, A. Kurkela, P. Romatschke, W. van der Schee, and Y. Zhu, Weak and strong coupling equilibration in nonabelian gauge theories, J. High Energy Phys. 04 (2016) 031.

[7] P. B. Arnold, G. D. Moore, and L. G. Yaffe, Transport coefficients in high temperature gauge theories. 2. Beyond leading log, J. High Energy Phys. 05 (2003) 051.

[8] M. A. York and G. D. Moore, Second order hydrodynamic coefficients from kinetic theory, Phys. Rev. D 79, 054011 (2009).

[9] A. Kurkela and E. Lu, Approach to Equilibrium in Weakly Coupled Non-Abelian Plasmas, Phys. Rev. Lett. 113, 182301 (2014).

[10] A. Kurkela and Y. Zhu, Isotropization and Hydrodynamization in Weakly Coupled Heavy-Ion Collisions, Phys. Rev. Lett. 115, 182301 (2015).

[11] T. Biro and J. Zimanyi, Quarkochemistry In Relativistic Heavy Ion Collisions, Phys. Lett. 113B, 6 (1982).

[12] J. Cleymans, I. Kraus, H. Oeschler, K. Redlich, and S. Wheaton, Statistical model predictions for particle ratios at $\sqrt{{ }^{s} \mathrm{NN}}=5.5 T e V$, Phys. Rev. C 74, 034903 (2006).

[13] A. Andronic, P. Braun-Munzinger, and J. Stachel, Thermal hadron production in relativistic nuclear collisions: The Hadron mass spectrum, the horn, and the QCD phase transition, Phys. Lett. B 673, 142 (2009); Erratum, Phys. Lett. B678, 516(E) (2009).

[14] A. Andronic, P. Braun-Munzinger, K. Redlich, and J. Stachel, Decoding the phase structure of QCD via particle production at high energy, Nature (London) 561, 321 (2018).

[15] G. Beuf, M. P. Heller, R. A. Janik, and R. Peschanski, Boost-invariant early time dynamics from AdS/CFT, J. High Energy Phys. 10 (2009) 043.

[16] P. M. Chesler and L. G. Yaffe, Holography and Colliding Gravitational Shock Waves in Asymptotically $\mathrm{AdS}_{5}$ Spacetime, Phys. Rev. Lett. 106, 021601 (2011).

[17] T. S. Biro, E. van Doorn, B. Muller, M. H. Thoma, and X. N. Wang, Parton equilibration in relativistic heavy ion collisions, Phys. Rev. C 48, 1275 (1993).

[18] G. Baym, Thermal equilibration in ultrarelativistic heavy ion collisions, Phys. Lett. B 138B, 18 (1984).

[19] K. Geiger and B. Muller, Dynamics of parton cascades in highly relativistic nuclear collisions, Nucl. Phys. B369, 600 (1992).
[20] B. Zhang, ZPC 1.0.1: A Parton cascade for ultrarelativistic heavy ion collisions, Comput. Phys. Commun. 109, 193 (1998).

[21] V. Borchers, J. Meyer, S. Gieseke, G. Martens, and C. C. Noack, A Poincare covariant parton cascade model for ultrarelativistic heavy ion reactions, Phys. Rev. C 62, 064903 (2000).

[22] Z. Xu and C. Greiner, Thermalization of gluons in ultrarelativistic heavy ion collisions by including three-body interactions in a parton cascade, Phys. Rev. C 71, 064901 (2005).

[23] J.-P. Blaizot, B. Wu, and L. Yan, Quark production, BoseEinstein condensates and thermalization of the quarkgluon plasma, Nucl. Phys. A930, 139 (2014).

[24] X.-G. Huang and J. Liao, Glasma evolution and BoseEinstein condensation with elastic and inelastic collisions, Phys. Rev. D 91, 116012 (2015).

[25] F. Scardina, D. Perricone, S. Plumari, M. Ruggieri, and V. Greco, Relativistic Boltzmann transport approach with Bose-Einstein statistics and the onset of gluon condensation, Phys. Rev. C 90, 054904 (2014).

[26] P. B. Arnold, G. D. Moore, and L. G. Yaffe, Effective kinetic theory for high temperature gauge theories, J. High Energy Phys. 01 (2003) 030.

[27] E. Braaten and R. D. Pisarski, Soft amplitudes in hot gauge theories: A general analysis, Nucl. Phys. B337, 569 (1990).

[28] L. D. Landau and I. Pomeranchuk, Electron cascade process at very high-energies, Dokl. Akad. Nauk Ser. Fiz. 92, 735 (1953).

[29] L. D. Landau and I. Pomeranchuk, Limits of applicability of the theory of bremsstrahlung electrons and pair production at high-energies, Dokl. Akad. Nauk Ser. Fiz. 92, 535 (1953).

[30] A. B. Migdal, Bremsstrahlung and pair production in condensed media at high-energies, Phys. Rev. 103, 1811 (1956).

[31] A. B. Migdal, Quantum kinetic equation for multiple scattering, Dokl. Akad. Nauk Ser. Fiz. 105, 77 (1955).

[32] M. Mace, S. Schlichting, and R. Venugopalan, Offequilibrium sphaleron transitions in the Glasma, Phys. Rev. D 93, 074036 (2016).

[33] L. Keegan, A. Kurkela, A. Mazeliauskas, and D. Teaney, Initial conditions for hydrodynamics from weakly coupled pre-equilibrium evolution, J. High Energy Phys. 08 (2016) 171.

[34] D. G. Figueroa, J. Garcia-Bellido, and F. Torrenti, Decay of the standard model Higgs field after inflation, Phys. Rev. D 92, 083511 (2015).

[35] T. Lappi and L. McLerran, Some features of the glasma, Nucl. Phys. A772, 200 (2006).

[36] F. Gelis, E. Iancu, J. Jalilian-Marian, and R. Venugopalan, The color glass condensate, Annu. Rev. Nucl. Part. Sci. 60, 463 (2010).

[37] M. C. A. York, A. Kurkela, E. Lu, and G. D. Moore, UV cascade in classical Yang-Mills theory via kinetic theory, Phys. Rev. D 89, 074036 (2014).

[38] J. Ghiglieri, G. D. Moore, and D. Teaney, Jet-medium interactions at NLO in a weakly-coupled quark-gluon plasma, J. High Energy Phys. 03 (2016) 095.

[39] D. Teaney (private communication). 
[40] P. Aurenche, F. Gelis, and H. Zaraket, A Simple sum rule for the thermal gluon spectral function and applications, J. High Energy Phys. 05 (2002) 043.

[41] J. Ghiglieri and G. D. Moore, Low mass thermal dilepton production at NLO in a weakly coupled quark-gluon plasma, J. High Energy Phys. 12 (2014) 029.

[42] A. Kurkela, A. Mazeliauskas, J.-F. Paquet, S. Schlichting, and D. Teaney, Effective kinetic description of event-byevent pre-equilibrium dynamics in high-energy heavy-ion collisions, arXiv:1805.00961 [Phys. Rev. C (to be published)].

[43] A. Kurkela, A. Mazeliauskas, Jean-Franois Paquet, S. Schlichting, and D. Teaney, Matching the non-equilibrium initial stage of heavy ion collisions to hydrodynamics with QCD kinetic theory, arXiv:1805.01604 [Phys. Rev. Lett. (to be published)].

[44] J. Ghiglieri, Guy D. Moore, and D. Teaney, QCD shear viscosity at (almost) NLO, J. High Energy Phys. 03 (2018) 179.

[45] J. Ghiglieri, G. D. Moore, and D. Teaney, Second-Order Hydrodynamics in Next-to-Leading-Order QCD, Phys. Rev. Lett. 121, 052302 (2018),

[46] J. Berges, S. Scheffler, and D. Sexty, Turbulence in nonabelian gauge theory, Phys. Lett. B 681, 362 (2009),

[47] J. Berges, S. Schlichting, and D. Sexty, Over-populated gauge fields on the lattice, Phys. Rev. D 86, 074006 (2012),

[48] S. Schlichting, Turbulent thermalization of weakly coupled non-Abelian plasmas, Phys. Rev. D 86, 065008 (2012),

[49] J. Berges, K. Boguslavski, S. Schlichting, and R. Venugopalan, Universal attractor in a highly occupied non-Abelian plasma, Phys. Rev. D 89, 114007 (2014),

[50] A. Kurkela and G. D. Moore, Thermalization in weakly coupled nonabelian plasmas, J. High Energy Phys. 12 (2011) 044.

[51] A. Kurkela and G. D. Moore, UV cascade in classical YangMills theory, Phys. Rev. D 86, 056008 (2012).

[52] D. Gelfand, F. Hebenstreit, and J. Berges, Early quark production and approach to chemical equilibrium, Phys. Rev. D 93, 085001 (2016).
[53] R. Baier, A. H. Mueller, D. Schiff, and D. T. Son, "Bottom up" thermalization in heavy ion collisions, Phys. Lett. B 502, 51 (2001).

[54] S. Mrowczynski, Stream instabilities of the quark-gluon plasma, Phys. Lett. B 214, 587 (1988); Erratum, Phys. Lett. B656, 273(E) (2007).

[55] S. Mrowczynski, Plasma instability at the initial stage of ultrarelativistic heavy ion collisions, Phys. Lett. B 314, 118 (1993).

[56] S. Mrowczynski and M.H. Thoma, Hard loop approach to anisotropic systems, Phys. Rev. D 62, 036011 (2000).

[57] A. Kurkela and G. D. Moore, Bjorken Flow, Plasma instabilities, and thermalization, J. High Energy Phys. 11 (2011) 120 .

[58] J. Berges, K. Boguslavski, S. Schlichting, and R. Venugopalan, Turbulent thermalization process in heavyion collisions at ultrarelativistic energies, Phys. Rev. D 89, 074011 (2014).

[59] S. Mrowczynski, Quasiquarks in two stream system, Phys. Rev. D 65, 117501 (2002).

[60] B. Schenke and M. Strickland, Fermionic collective modes of an anisotropic quark-gluon plasma, Phys. Rev. D 74, 065004 (2006).

[61] T. Lappi, Gluon spectrum in the glasma from JIMWLK evolution, Phys. Lett. B 703, 325 (2011).

[62] H. Kouno, M. Maruyama, F. Takagi, and K. Saito, Relativistic hydrodynamics of quark-gluon plasma and stability of scaling solutions, Phys. Rev. D 41, 2903 (1990).

[63] A. Muronga, Second Order Dissipative Fluid Dynamics for Ultrarelativistic Nuclear Collisions, Phys. Rev. Lett. 88, 062302 (2002); 89, 159901(E) (2002).

[64] E. V. Shuryak, Two Stage Equilibration in HighEnergy Heavy Ion Collisions, Phys. Rev. Lett. 68, 3270 (1992).

[65] A. Kurkela and A. Mazeliauskas, Chemical equilibration in hadronic collisions, arXiv:1811.03040 [Phys. Rev. Lett. (to be published)]. 